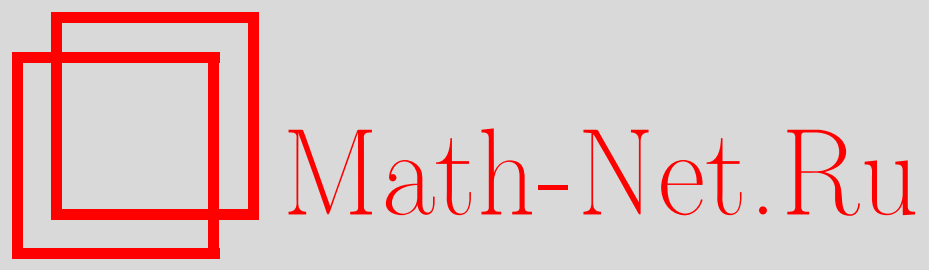

А. С. Осипов, Об интегрировании неабелевых цепочек типа Ленгмюра методом обратной спектральной задачи, Функи. анализ и его прил., 1997, том 31, выпуск 1, 86-89

DOI: https://doi.org/10.4213/faa452

Использование Общероссийского математического портала MathNet.Ru подразумевает, что вы прочитали и согласны с пользовательским соглашением http://www . mathnet.ru/rus/agreement

Параметры загрузки:

IP : 54.89 .56 .158

26 апреля 2023 г., 14:06:38

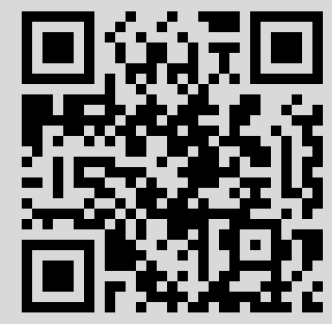


с точностью до растяжения (т.е. умножения на элемент группы $\mathbb{Q}_{p}^{*}$ ). Иными словами, $\mathrm{Ens}_{n}$ - множество вершин ансамбля Брюа-Титса.

Рассмотрим теперь пространство $A=M / \mathbb{Q}_{p}^{*}$, а в качестве $\mathscr{A}$ возьмем множество $\mathrm{Ens}_{n}$. Тогда допустимые подмножества в $M / \mathbb{Q}_{p}^{*}$ имеют вид

$$
0=M_{0}, L_{0}, M_{1}, L_{1}, M_{2}, L_{2}, \ldots, M_{k+1}=\mathbb{Q}_{p}^{n},
$$

где $M_{0} \subset M_{1} \subset \cdots \subset M_{k+1}$ - линейные подпространства в $\mathbb{Q}_{p}^{n}$ и $M_{j} \subset L_{j} \subset$ $M_{j+1}$, причем $L_{j} / M_{j}$ - решетки в $M_{j+1} / M_{j}$.

Таким образом, множество $\mathrm{Ens}_{n}$ естественным образом компактифицируется пространством допустимых подмножеств в $A=M / \mathbb{Q}_{p}^{*}$.

Я благодарю К. Де Кончини и С. Л. Трегуба за обсуждение затронутой темы. Я также благодарю руководство Института Макса Планка (Бонн) за гостеприимство.

\title{
ЛитеРАТУРА
}

1. Pompeiu D. Ann. Fac. Sci. Toulouse, 7, 265-315 (1905). 2. Хаусдор $\Phi$ Ф. Теория множеств. ОНТИ, 1937. 3. Study E. Math. Ann., 27, 51-58 (1886). 4. Semple I. G. Rend. Math. Univ. Roma (5), 10, 201-208 (1951). 5. Satake I. Ann. Math., 77, 335-386 (1963). 6. Alguineid A. R. Proc. Math. Phys. Soc. Eqypt, 4, 93-104 (1952). 7. Furstenberg H. Ann. Math., 77, 335-386 (1963). 8. De Concini C., Procesi C. Lect. Notes Math., vol. 996, 1983, pp. 1-44. 9. Неретин Ю. А. Функц. анализ и его прил., 26, вып. 4, 30-44 (1992). 10. Neretin Yu. A. Preprint MPI 96-78. 11. Kapranov M. M. Adv. Sov. Math., Vol. 16, Part 2, 29-110. 12. Neretin Yu. A. To appear in Kirillov seminar on representation theory, Adv. Sov. Math.

Московский государственный институт электроники и математики,

Max-Planck-Institut für Mathematik (Bonn)

Поступило в редакцию 24 апреля 1996 г.

\section{Об интегрировании неабелевых цепочек типа Ленгмюра методом обратной спектральной задачи}

\author{
(c) 1997. А. С. Осипов
}

Рассматривается задача Коши для системы дифференциально-разностных уравнений, коэффициенты которых - ограниченные операторы из произвольного банахова пространства $B$ :

$$
\begin{gathered}
\dot{C}_{n}=\sum_{i=1}^{q} C_{n+i} C_{n}-C_{n} \sum_{i=1}^{q} C_{n-i}, \quad q \in \mathbb{N}, \\
C_{n}=C_{n}(t) \in \mathfrak{L}(B), \quad t \in[0, T), \quad 0<T \leqslant \infty, \quad \cdot=d / d t .
\end{gathered}
$$

В скалярном случае при $C_{n}(t)>0$ и $q=1$ исследуемая система представляет собой известную в приложениях цепочку Ленгмюра (модель Вольтерра), интегрируемую различными методами обратной задачи $[1,2]$. Для произвольных $q$ 
система (1) с числовыми коэффициентами исследовалась в $[3,4]$, причем в [3] было установлено, что она является дискретным аналогом уравнения Кортевегаде Фриза. При $q=1$ данная система в полубесконечном случае $\left(n \in \mathbb{Z}_{+}\right)$была рассмотрена в [5]. Ниже будут приведены процедуры построения ограниченных решений системы (1) как для полубесконечного, так и для бесконечного $(n \in \mathbb{Z})$ случая. При этом потребуется обобщить метод обратной спектральной задачи для несимметрических разностных операторов второго порядка [5] на некоторые классы разностных операторов высших порядков.

1. Полубесконечный случай. Система (1) допускает представление Лакса $\dot{L}=[L, A]$, где $L=L(t)=\left(L_{i j}\right)_{i, j=0}^{\infty}$ и $A=A(t)=\left(A_{i j}\right)_{i, j=0}^{\infty}$ - матрицы, ненулевые элементы которых имеют вид

$$
\begin{gathered}
L_{i, i-q}=C_{i-q}, \quad L_{i, i+1}=E \quad(E-\text { тождественный оператор }), \\
A_{i, i}=-C_{i-q}-\cdots-C_{i}, \quad A_{i, i+q+1}=-E, \quad i \geqslant 0 .
\end{gathered}
$$

Будем искать решения системы (1), удовлетворяющие условиям

$$
\sup _{n \in \mathbb{Z}_{+}, t \in[0, T)}\left(\left\|C_{n}(t)\right\|\right)<\infty, \quad C_{n}(t) \text { обратимы. }
$$

Тогда при всех $t$ матрищы $L(t)$ и $A(t)$ порождают ограниченные операторы в пространстве $l_{1}([0, \infty), B)=\left\{\left(u_{n}\right)_{n=0}^{\infty} ; u_{n} \in B, \sum_{n=0}^{\infty}\left\|u_{n}\right\|<\infty\right\}$, за которыми мы сохраним те же обозначения.

Для оператора $L=L(t)$ введем матрицу Вейля $\mathfrak{M}(\lambda)=\mathfrak{M}(\lambda, t)$ :

$$
\mathfrak{M}(\lambda)=\left(\phi_{1}(\lambda), \ldots, \phi_{q}(\lambda)\right),
$$

где $\phi_{i}(\lambda)=\left(R_{\lambda} e_{i}\right)_{0}, e_{i}=\left(\delta_{i-1, k} E\right)_{k=0}^{\infty}, R_{\lambda}=(\lambda E-L(t))^{-1}, i=1, \ldots, q$. Следовательно,

$$
\phi_{i}(\lambda)=\sum_{k=0}^{\infty} \frac{S_{k}^{i}}{\lambda^{k+1}}, \quad \text { где } S_{k}^{i}=\left(L^{k}\right)_{0, i-1} .
$$

Можно рассматривать $\mathfrak{M}(\lambda)$ для оператора $L$ как обобщение функции Вейля для полубесконечных матрищ Якоби.

Будем называть последовательность $S(\mathfrak{M}(\lambda))=\left(S_{k}^{1}, \ldots, S_{k}^{q}\right)_{k=0}^{\infty}$ моментной последовательностью матрицы Вейля, а $S_{k}^{i}$ - ее моментами. Следующая теорема дает критерий разрешимости обратной задачи для оператора $L(t)$ и алгоритм восстановления его элементов по $S(\mathfrak{M}(\lambda))$.

Теорема 1. Для того чтоби последовательность $\left(S_{k}^{1}, \ldots, S_{k}^{q}\right)_{k=0}^{\infty}, S_{k}^{i} \in$ $\mathfrak{L}(B)$, такая, что степенные ряды, построенные по формулам (5), являются голоморфньми в бесконечности оператор-функииями, была моментной последовательностью матриць Вейля оператора $L$, удовлетворяюшего условиям (2)-(4), необходимо и достаточно вьполнение таких условий:

(i) $S_{i-1}^{i}=E, i=1, \ldots, q$;

(ii) $S_{n+(1+q) p}^{i}=0, p \in \mathbb{Z}_{+}, n=0, \ldots, q, n \neq i-1$;

(iii) $n р u$ любом $k \geqslant 0$ операторы $\Delta_{k}=\left(\alpha_{r j}\right)_{r, j=0}^{k}$, 2де $\alpha_{r j}=S_{j+p+1}^{l+1} n p u$ $r=p q+l, p \in \mathbb{Z}_{+}, l=0, \ldots, q-1$, обратимьи в пространстве $B^{k}=$ $\left\{\left(u_{j}\right)_{j=0}^{k} ; u_{j} \in B\right\}$. 
Коэффичиенты оператора $L$ восстанавливаются посредством следуюшей прочедурь:

(a) по последовательности $S(\mathfrak{M}(\lambda))=S^{0}(\mathfrak{M}(\lambda))$ рекуррентно строится последовательность $S^{n}(\mathfrak{M}(\lambda))=\left(S_{k}^{1, n}, \ldots, S_{k}^{q, n}\right)_{k=0}^{\infty}, n \in \mathbb{Z}_{+}$:

$$
S_{k}^{i, n+1}=D_{k}^{i+1}\left(S^{n}\right), \quad i=1, \ldots, q-1, \quad S_{k}^{q, n+1}=\left(D_{k+1}^{1}\left(S^{n}\right)\right)\left(S_{q+1}^{1, n}\right)^{-1},
$$

$k \in \mathbb{N}$, где

$$
\begin{aligned}
D_{k}^{1}\left(S^{n}\right) & =\sum_{i_{1}+\cdots+i_{m}=k+1}(-1)^{m+1} S_{i_{1}}^{1, n} \cdots S_{i_{m}}^{1, n}, \\
D_{k}^{i+1}\left(S^{n}\right) & =\sum_{i_{1}+\cdots+i_{m}=k-p}(-1)^{m} S_{i_{1}}^{1, n} \cdots S_{i_{m}}^{1, n} \sum_{p=0}^{k-1} S_{p+1}^{i+1, n}+S_{k+1}^{i+1, n},
\end{aligned}
$$

$i_{1}, \ldots, i_{m} \geqslant 1$ и суммирование ведется по всем наборам $i_{1}, \ldots, i_{m}$, удовлетворлющим указанньм равенствам, $а S_{0}^{i, n+1}=\delta_{i-1,0} E, i=1, \ldots, q$;

(b) по $S^{n}(\mathfrak{M}(\lambda))$ определяются коэффичиенты оператора $L$ посредством формульь

$$
C_{n}=L_{n+q, n}=S_{q+1}^{1, n} .
$$

Отметим, что условие (ii) теоремы 1 является критерием выполнения следующего условия для оператора $L: L_{i, i+j}=0, i \geqslant 0, j=-q+1, \ldots, 0$.

Используя представление Лакса для системы (1) в предположении, что ее решение сушествует, получаем эволюционные уравнения для моментов $S_{k}^{i}(t)$ :

$$
\dot{S}_{k}^{i}(t)=S_{k+q+1}^{i}(t)-S_{k}^{i}(t) S_{i+q+1}^{i}(t), \quad i=1, \ldots, q .
$$

Основная теорема для полубесконечного случая формулируется следуюшим обра3ом.

Теорема 2. Пусть для системь (1) начальные даннье $\left(C_{n}(0)\right)_{n=0}^{\infty}$ удовлетворлют (4). Тогда найдется $\delta>0$, такое, что при $t \in[0, \delta)$ решение задачи Коши для (1), удовлетворлющее (4), существует, единственно и строится следуюшим образом:

(i) по формулам (2) определяется оператор $L(0)$;

(ii) используя (5), для $L(0)$ строится моментная последовательность матричь Вейля $S(\mathfrak{M}(\lambda, 0))=\left(S_{k}^{1}(0), \ldots, S_{k}^{q}(0)\right)_{k=0}^{\infty} ;$

(iii) определяется последовательность $S(\mathfrak{M}(\lambda, t))=\left(S_{k}^{1}(t), \ldots, S_{k}^{q}(t)\right)_{k=0}^{\infty}$ :

$$
S_{k}^{i}(t)=\left(\sum_{d=0}^{\infty} S_{k+(q+1) d}^{i}(0) \frac{t^{d}}{d !}\right)\left(\sum_{d=0}^{\infty} S_{i+(q+1) d}^{i}(0) \frac{t^{d}}{d !}\right)^{-1}, \quad i=1, \ldots, q ;
$$

(iv) nо $S(\mathfrak{M}(\lambda, t))$, исходя из (6), (7), вычисляяются $C_{n}(t)$,

Если для построенной последовательности $S(\mathfrak{M}(\lambda, t))$ условие (iii) теоремь 1 выполнено при $t \in[0, T)$, то указанная прочедура дает решение системь на всем рассматриваемом интервале.

2. Бесконечный случай. Здесь можно получить решения системы (1) в виде степенных рядов, коэффициенты которых строятся при помощи конечных систем, аппроксимирующих (1). Этот метод использовался в [6] при интегрировании бесконечной цепочки Тода. А именно справедлива следующая теорема: 
ТЕОРЕМА 3. Пусть начальнье даннье для (1) удовлетворяют условию $\left\|C_{n}(0)\right\| \leqslant K \quad$ u $C_{n}(0)$ обратимьи, $n \in \mathbb{Z}$. Тогда существует $\delta=\delta(K)>0$, такое, что при $t \in[0, T)$ решение задачи Коши для (1) существует, единственно и представимо в виде ряда

$$
C_{n}(t)=\sum_{p=0}^{\infty} \frac{C_{n p} t^{p}}{p !}, \quad n \in \mathbb{Z} .
$$

Коэффициентьи $C_{n р}$ определяются следуюшим образом:

(i) строится конечная матрица $L(n, p, 0)=\left(L(n, p, 0)_{i j}\right)_{i, j=0}^{q(2 p+1)}$ с ненулевыми элементами

$$
L(n, p, 0)_{i, i-q}=C_{n-p q+i-q}(0), \quad L(n, p, 0)_{i, i+1}=E, \quad i=0, \ldots, q(2 p+1) ;
$$

(ii) определяется моментная последовательность $S\left(\mathfrak{M}_{p}(\lambda, 0)\right)=\left(S_{k, p}^{1}(0)\right.$, $\left.\ldots, S_{k, p}^{q}(0)\right)_{k=0}^{\infty}$ по формуле $S_{k, p}^{i}=\left(L^{k}(n, p, 0)\right)_{0, i-1}, i=1, \ldots, q$;

(iii) при помощи формул (8) теоремь 2 и (6) теоремь 1 строится последовательность $\left(S_{k, p}^{1, l}(t), \ldots, S_{k, p}^{q, l}(t)\right)_{k=0}^{\infty}=S^{l}\left(\mathfrak{M}_{p}(\lambda, t)\right), l \in \mathbb{Z}_{+} ;$

(iv) вьчисляются $C_{n p}$ по формулам

$$
C_{n p}=\left.\frac{d^{p}}{d t^{p}}\left(S_{q+1, p}^{1, q p}(t)\right)\right|_{t=0} .
$$

Автор благодарен А. Г. Костюченко за внимание к работе.

$$
\text { ЛитЕРАТУРА }
$$

1. Kac M., van Moerbeke P. Adv. Math., 16, 160-169 (1975). 2. Berezansky Yu. M. Rep. Math. Phys., 24, No. 1, 21-47 (1986). 3. Богоявленский О. И. Изв. АН СССР, cер. матем., 51, №6, 1123-1142 (1987). 4. Itoh I. Progr. Theoret. Phys., 7, No. 3, 507-510 (1987). 5. Гехтман М. И. Функц. анализ и его прил., 24, вып. 3, 72-73 (1990). 6. Гехтман М. И. Функц. анализ и его прил., 25, вып. 3, 82-84 (1991).

Институт системных исследований РАН

Поступило в редакцию 22 ноября 1995 г.

УДК 519.46

\section{Минимальная система определяющих соотношений для некоторых супералгебр Ли}

(C) 1997. Г.И. Просветов

В данной работе найдены минимальные системы определяюших соотношений (т.е. минимальные системы соотношений между образующими) для некоторых супералгебр Ли.

Для алгебры Каца-Муди $L(A)$ с обобщенной $n \times n$-матрицей Картана $A=$ $\left(a_{i j}\right)$ и образующими Шевалле $e_{i}, f_{i}(i=1, \ldots, n)$ имеется треугольное разложение $L(A)=L_{-} \oplus L_{0} \oplus L_{+}$. Здесь $L_{+}$(соответственно $\left.L_{-}\right)$обозначает подалгебру в $L(A)$, порожденную элементами $e_{1}, \ldots, e_{n}\left(\right.$ соответственно $\left.f_{1}, \ldots, f_{n}\right)$, а 